\title{
Changes in Co-Occurrence of Smoking and Harmful Drinking among Youth: a Study from the Chi Linh Demographic - Epidemiological Surveillance System in Vietnam, 2006-2013
}

\author{
Duong Minh Duc ${ }^{1,2 *}$, Le Thi Vui ${ }^{1}$, Nguyen Thuy Quynh ${ }^{1}$, Hoang Van Minh ${ }^{1}$
}

\begin{abstract}
Smoking and harmful drinking dramatically increase health risks but little is known about their cooccurrence and factors that influence this co-habit, limiting development and implementation of appropriately targeted prevention interventions. This study was conducted among youth aged 10-24 years old in the Chi Linh Demographic - Epidemiological Surveillance System (CHILILAB DESS). The total numbers in the first, second and third rounds in 2006, 2009 and 2013 were 12,406, 10,211, and 7,654, respectively. A random-effects logit model controlling for both time-variant and time-invariant variables was applied to explore factors associated with current smoking, harmful drinking, and occurrence of smoking and harmful drinking together. We found dramatically increasing trends in current smoking, harmful drinking and co-occurrence among youth. Our results indicate similar health problems among youth in peri-urban areas in Vietnam. Demographic characteristics (older age, being male, being unmarried, and having informal work) appeared to be predictors for smoking and drinking behaviour. Besides, peer and family members had significant influence on smoking, whereas having a close-friend who was smoking was the most important variable. The results suggested that smoking and harmful drinking should not be solved with separate, stand-alone interventions but rather with integrated efforts.
\end{abstract}

Keywords: Smoking - harmful drinking - co-occurrence - youth - Viet Nam - CHILILAB

Asian Pac J Cancer Prev, 17 Tobacco Prevention and Control in Vietnam Suppl, 55-63

\section{Introduction}

The co-occurrence of smoking and drinking, particularly young adults who are beginning to experiment with smoking, has been shown in many studies (Johnson et al., 2000; Dierker et al., 2006; Jackson et al., 2010). Numerous studies have demonstrated a positive association between smoking and drinking (Schmid et al., 2007; Witkiewitz et al., 2012). Youths in United States, for example, drink significantly more drinks per day when smoking and smoke significantly more cigarettes when drinking (Jackson et al., 2010). Using a nationally representative sample, a study in the US found $98 \%$ of student smokers drank alcohol (Weitzman and Chen, 2005). Smokers are much more likely to use alcohol and drinkers are much more likely to smoke (Johnson et al., 2000; Weitzman and Chen, 2005)). The co-occurrence of smoking and drinking is a major concern because of the dramatically increase health risks such as higher risk for certain types of cancer, particularly those of the mouth and throat (Johnson et al., 2000; Weitzman and Chen, 2005).

Although a number of studies highlight the significant increase co-occurrence of smoking and drinking, little is known about the patterns of smoking and drinking among adolescents and youth in low- and middle-income countries (LMICs). In recent years, adult co-occurring smoking and drinking has been urged as the first most important risk factor for ill-health and premature death in LMICs (Anderson, 2006). Similar to other LMICs in the Asian region, Vietnam has very little evidence about patterns of adolescent co-occurrence of smoking and drinking and how certain demographic subgroups of adolescents are similar to, or differ from others. Moreover, few publications have offered evidence to predict the co-occurring smoking and drinking among youth in Vietnam. Some recent studies report that certain subgroups of adolescents have much higher rates of smoking and drinking (Giang et al., 2008; Guindon et al., 2008; Hoang et al., 2010). The male smoking rate ratio, for example, is 10 times higher than women smoking rate, men also smoking significantly more cigarettes per day than women (Giang et al., 2008; Guindon et al., 2008; Hoang et al., 2010). There is, however, growing concern about changes in patterns of smoking and drinking among youth in Vietnam (Nguyen et al., 2012).

Society's treatment of smoking and drinking is complex and has radically changed over the years in Vietnam. Smokers, for example, are forced to stand isolated outside of public places (Bui et al., 2015). A number of different measures have been implemented in

${ }^{1}$ Hanoi School of Public Health, Ha Noi, Vietnam, ${ }^{2}$ International Maternal and Child Health (IMCH), Department of Women's and Children's Health, Uppsala University, Uppsala, Sweden*For correspondence:dmd@hsph.edu.vn 
tobacco and alcohol control programmes to reduce the prevalence of use in Vietnam (Bui et al., 2015). There has, however, an increased risk of co-occurring smoking and drinking among some sub-groups of youth in Vietnam (Nguyen et al., 2012). Many studies have provided a better understanding of the antecedents of engaging in smoking or drinking among youths. Few studies, however, have examined the specific factors that influence cooccurring smoking and drinking among youth in LMICs. Understanding the factors that predict co-occurring smoking and drinking may provide valuable information for efforts to reduce smoking-related and drinking-related morbidity and mortality. Therefore, we aimed to examine the changes in prevalence of co-occurring smoking and harmful drinking among youth and identify factors which are significantly associated with this behaviour in a periurban area in Vietnam during 2006-2013.

\section{Materials and Methods}

\section{Study setting}

The study was conducted in the Chi Linh Demographic - Epidemiological Surveillance System (CHILILAB DESS) in Chi Linh, a peri-urban district in a northern province in Vietnam. This district covers an area of about 300 square kilometres including 12 communes and 8 towns with about 175,000 residents by 2015 (General Statistic Office of Vietnam, 2015). The population density varies with a higher density in lowland communes. Approximately a fourth of the total population lives in urban areas.

The CHILILAB provides data on an urban and rural population, which is in view of the rapid economic and population growth in Vietnam. The CHILILAB is one of the few field laboratories in the world that conduct both operational and intervention research with field training (Tran et al., 2013). In addition to collecting quarterly basic health and demographic data, CHILILAB was designed to collect data on adolescent health. The adolescent health survey, which was conducted in 7 communes/towns in Chi Linh, to examine comprehensive situational assessment to better understand the current situation in Vietnam with regard to youth health, associated behaviours and the related antecedents that both predispose to risk and those that diminish health compromising behaviours.

\section{Study design and population}

This was a longitudinal cohort study among all youth aged 10-24 years old in 7 communes/towns in Chi Linh. Data were collected in three rounds during 7 years (20062013) at 3-year intervals from the same adolescents. Youth, who did not participate in three rounds, were either sick or did not stay in their house during the survey. The first, second, and third rounds of the survey were conducted during July 2006-January 2007, February-July 2009, and September-December 2013, respectively. The duration between the second and third round was longer than the duration between the first and second round due to a delay in survey organization. Virtually all youth had 7 years between the first and last survey. The three rounds included information from the household survey, for example, sex, education level, household quintile economy using DESS questionnaires. The household survey, which has been conducted biennially at both the household and individual levels since 2004, covered approximately 18,000 households in Chi Linh.

\section{Data collection}

The current study was based on existing adolescent health survey data. The survey included three surveys, that is, health status and behaviour survey, risk and protective factors survey, and parent/family survey. The health status and behaviour survey is used for the analysis in this paper to obtain an accurate picture of the current health status of youth, experience with adverse health-related events, and risk-taking and health-seeking behaviours.

Data collection was done by trained female interviewers, who were also the data collectors of the CHILILAB DESS. They were local residents who had been selected by a thorough recruitment process. Most of these data collectors participated in all three rounds of survey as the turnover rate was as low as $10 \%$. Several measures were conducted to ensure the quality of the data. A separate quality-control re-surveyed $5 \%$ of the households and the main survey data were cross-checked with the findings to validate data. Differences were rechecked and discussed to reach consensus.

The total numbers of youths in the first, second, and third rounds were $12,406,10,211$, and 7,654, respectively. One-third of youths were excluded due to loss of follow-up in either the second or the third round.

\section{Study variables}

Outcome variables: To assess current smoking, youth were asked how often, if ever, they had used cigarettes. The answers were then categorized to 'never used' and 'used in the last 30 days'. Current smoking was defined as those who reported to have used cigarettes in the past 30 days. Harmful drinking behaviour was measured using the question, 'Have you ever gotten into trouble because of drinking'. The answers included (1) got trouble with parents; (2) got trouble at school; (3) got trouble with friends; (4) doing something regretted; (5) got dizzy and vomit; (6) got stomach ache; (7) having unwanted sex; and (8) got into a physical fight. These responses were then categorized into 'no' and 'ever had trouble' (for example, physical harm or having unwanted sex). Harmful drinking was defined as those who reported that they had gotten into trouble because of drinking. Co-occurrence of smoking and harmful drinking behaviour was constructed as a binary variable that reflected whether the respondent encountered both current smoking and harmful drinking.

Independent variables: We selected and divided 10 potential independent variables relating to smoking and drinking into two classes as follows: demographic variables (sex, age, marital status, occupation, household income, educational level, and setting) and family member and peer influences (family members' smoking, closefriends' smoking, and family members' drinking). All these potential variables were evaluated in the present study. 
Changes in Co-Occurrence of Smoking and Harmful Drinking among Youth from Chi Linh in Vietnam, 2006-2013

Sex was composed of two categories: male and female. Age was categorised into three age groups of 10-14, 1519, and 20-24 years. Marital status was dichotomised into married or single and the single group included those who were divorced. Household income was divided into two groups: poor and near poor (households that earn less than 1000 USD annually) and middle or higher income (households that earn more than 1000 USD annually). Current occupation was comprised of three groups: informal work (farmer, housework, and craft worker), student (students and vocational/work training) and others. Educational level was divided into two categories in the order of increasing level of education: high-school graduation or below and university degree or above. Setting was categorised as: rural and urban areas.

In terms of family member and peer influences, we asked about the cigarette smoking or alcohol drinking habits of the family members and their close-friends. We defined the smoking or drinking status as either 'yes' or 'no'. Having a close-friend who was currently smoking, for example, was defined if the respondent answered 'yes' for the question 'Is any of your close-friends smoking?'.

\section{Data analysis}

The data analyses were carried out in two regression models using Stata version 13. The primary model was to explore the empirical relationship between independent variables and the outcome measures among young people aged 15-24 years old using the logistic regression model per round. The final model which used random-effects logit model for panel data were computed to control for both time-variant and time-invariant variables in all three rounds. The rationale for using random-effects model is that the time-invariant characteristics, for example, gender and setting, have influences on the dependent variable. Simultaneously, the model could include the time-variant variables, such as, occupation and close-friends' smoking, which could be at-risk of co-occurring smoking and drinking. Thus, variables included in our analysis were dependent variable (current smoking, harmful drinking, and occurrence of smoking and harmful drinking), and independent variables (sex, age, marital status, occupation, household income, educational level, family members' smoking, close-friends' smoking, and family members' drinking). We also included current smoking or harmful drinking when undertaking our models to predict harmful drinking or smoking habit, respectively. P-value $<0.05$ was considered significant.

\section{Ethical considerations}

Ethical approval for this study was obtained from the Institutional Review Board of Ha Noi School of Public Health. All respondents were asked to participate in the survey by signing informed consent document. Further caregivers' agreement was also collected if the adolescents were under 18 years of age. Respondents could refuse to participate in or withdraw from the interview at any time. Respondents had been given a small present for their participation.

\section{Results}

General characteristics of the study respondents

The total sample size was lower after each round, ranging from a high of 12,406 in 2006 to a low of 7,654 in 2013 (see Table 1). The mean age of the sample by 2006

Table 1. Demographic Characteristics of Respondents: Three Survey Rounds 2006-2013

\begin{tabular}{|c|c|c|c|c|c|c|c|}
\hline & \multirow{2}{*}{ Characteristics $(\mathrm{N}, \%)$} & \multicolumn{2}{|c|}{2006} & \multicolumn{2}{|c|}{2009} & \multicolumn{2}{|c|}{2013} \\
\hline & & $\mathrm{n}$ & $\%$ & $\mathrm{n}$ & $\%$ & $\mathrm{n}$ & $\%$ \\
\hline & Overall & 12,406 & & 10,211 & & 7,654 & \\
\hline & \multicolumn{7}{|l|}{ Sex } \\
\hline \multirow[t]{3}{*}{1} & Male & 6,091 & 49.1 & 4,996 & 48.9 & 3,678 & 48.1 \\
\hline & Female & 6,315 & 50.9 & 5,215 & 51.1 & 3,976 & 52 \\
\hline & Age & & & & & & \\
\hline \multirow{3}{*}{2} & $10-14$ years old & 4,237 & 34.2 & 4,024 & 39.4 & 3,067 & 40.1 \\
\hline & $15-19$ years old & 5,047 & 40.7 & 3,884 & 38 & 2,776 & 36.3 \\
\hline & 20-24 years old & 3,122 & 25.2 & 2,303 & 22.6 & 1,811 & 23.7 \\
\hline \multirow{4}{*}{3} & Marital status & & & & & & \\
\hline & Unmarried & 11,339 & 91.4 & 8,420 & 84.4 & 5,509 & 72.8 \\
\hline & Married & 1,067 & 8.6 & 1,553 & 15.6 & 2,059 & 27.2 \\
\hline & Occupation & & & & & & \\
\hline \multirow{3}{*}{4} & Informal work & 1,859 & 15.2 & 1,413 & 13.8 & 1,177 & 15.4 \\
\hline & Pupils/ Students & 8,678 & 70.9 & 6,736 & 66 & 3,193 & 41.7 \\
\hline & Others & 1,708 & 14 & 2,061 & 20.2 & 3,284 & 42.9 \\
\hline \multirow{3}{*}{5} & Household income & & & & & & \\
\hline & Poor and near poor & 3,393 & 27.8 & 2,815 & 35.8 & 1,633 & 38.2 \\
\hline & Middle or higher income & 8,794 & 72.2 & 5,040 & 64.2 & 2,643 & 61.8 \\
\hline \multirow{3}{*}{6} & Education level & & & & & & \\
\hline & High school or lower level & 10,876 & 89.4 & 8,510 & 83.4 & 4,431 & 57.9 \\
\hline & College/ university degree or higher & 1,284 & 10.6 & 1,700 & 16.7 & 3,223 & 42.1 \\
\hline \multirow{3}{*}{7} & Setting & & & & & & \\
\hline & Rural & 6,397 & 51.9 & 5,291 & 51.8 & 4,018 & 53.9 \\
\hline & Urban & 5,925 & 48.1 & 4,920 & 48.2 & 3,433 & 46.1 \\
\hline
\end{tabular}


Table 2. Percentage Distribution of Smoking and Harmful Drinking Patterns, by Demographic Characteristics: Three Rounds of Survey 2006-2013

\begin{tabular}{|c|c|c|c|c|c|c|c|c|c|c|}
\hline \multirow{2}{*}{\multicolumn{2}{|c|}{$\begin{array}{c}\text { Variable }(\mathrm{N}, \%) \\
\text { Characteristics/ Year }\end{array}$}} & \multicolumn{3}{|c|}{ Current smoking } & \multicolumn{3}{|c|}{ Harmful drinking } & \multicolumn{3}{|c|}{$\begin{array}{l}\text { Co-occurrence of smoking } \\
\text { and harmful drinking }\end{array}$} \\
\hline & & 2006 & 2009 & 2013 & 2006 & 2009 & 2013 & 2006 & 2009 & 2013 \\
\hline \multirow{2}{*}{\multicolumn{2}{|c|}{ Overall }} & 1,350 & 1,304 & 1,279 & 2,783 & 2,735 & 2,019 & 818 & 812 & 703 \\
\hline & & 11.2 & 13.6 & 17.6 & 24.7 & 27.5 & 26.8 & 7.1 & 8.3 & 9.5 \\
\hline \multicolumn{2}{|r|}{ Sex } & & & & & & & & & \\
\hline \multirow{4}{*}{1} & \multirow{2}{*}{ Male } & 1,274 & 1,285 & 1,228 & 1,930 & 1,979 & 1,476 & 793 & 802 & 676 \\
\hline & & 21.5 & 28 & 36.3 & 36.1 & 41.4 & 41.3 & 13.8 & 17.2 & 19.4 \\
\hline & \multirow{2}{*}{ Female } & 76 & 19 & 51 & 853 & 756 & 543 & 25 & 10 & 27 \\
\hline & & 1.3 & 0.4 & 1.3 & 14.4 & 14.6 & 13.7 & 0.4 & 0.2 & 0.7 \\
\hline \multirow{7}{*}{2} & \multicolumn{10}{|l|}{ Age } \\
\hline & \multirow{2}{*}{$10-14$ years old } & 93 & 149 & 350 & 485 & 680 & 781 & 29 & 81 & 187 \\
\hline & & 2.2 & 3.9 & 12 & 12.1 & 17.1 & 25.7 & 0.7 & 2.1 & 6.3 \\
\hline & \multirow{2}{*}{ 15-19 years old } & 549 & 609 & 507 & 1,307 & 1,329 & 784 & 334 & 394 & 270 \\
\hline & & 11.2 & 16.9 & 19.3 & 28.7 & 35.2 & 28.8 & 6.9 & 10.7 & 10.1 \\
\hline & \multirow{2}{*}{$20-24$ years old } & 708 & 546 & 422 & 991 & 726 & 454 & 455 & 337 & 246 \\
\hline & & 23.8 & 24.9 & 24.6 & 36.6 & 33 & 25.6 & 15.7 & 15.5 & 14 \\
\hline \multirow{5}{*}{3} & \multicolumn{10}{|l|}{ Marital status } \\
\hline & \multirow{2}{*}{ Unmmarried } & 1,219 & 1,051 & 856 & 2,572 & 2,358 & 1,535 & 744 & 662 & 459 \\
\hline & & 11.1 & 13.3 & 16.4 & 25.1 & 28.7 & 28.3 & 6.8 & 8.2 & 8.6 \\
\hline & \multirow{2}{*}{ Married } & 131 & 242 & 417 & 211 & 331 & 459 & 74 & 144 & 241 \\
\hline & & 12.7 & 16.2 & 21.2 & 20.8 & 22 & 22.7 & 7.2 & 9.6 & 12 \\
\hline \multirow{7}{*}{4} & \multicolumn{10}{|l|}{ Occupation } \\
\hline & \multirow{2}{*}{ Informal work } & 444 & 414 & 357 & 564 & 436 & 346 & 273 & 256 & 209 \\
\hline & & 25 & 30.7 & 32 & 34 & 32.5 & 29.9 & 15.7 & 19.2 & 18.4 \\
\hline & \multirow{2}{*}{ Pupils/ Students } & 537 & 469 & 254 & 1,664 & 1,673 & 798 & 323 & 298 & 134 \\
\hline & & 6.4 & 7.5 & 8.4 & 20.9 & 25.2 & 25.2 & 3.8 & 4.6 & 4.3 \\
\hline & & 342 & 421 & 668 & 510 & 625 & 875 & 202 & 258 & 360 \\
\hline & Others & 20.9 & 21.5 & 21.4 & 34.3 & 31.4 & 27.2 & 12.7 & 13.2 & 11.3 \\
\hline & Household income & & & & & & & & & \\
\hline & & 417 & 365 & 349 & 730 & 672 & 456 & 242 & 225 & 192 \\
\hline 5 & Poor and near poor & 12.8 & 13.7 & 22.3 & 23.5 & 24.6 & 28.3 & 7.5 & 8.3 & 12.1 \\
\hline & & 913 & 553 & 394 & 2,007 & 1,259 & 662 & 565 & 347 & 212 \\
\hline & Middle or higher income & 10.7 & 11.6 & 15.8 & 25.2 & 25.5 & 25.4 & 6.6 & 7.2 & 8.3 \\
\hline & Education level & & & & & & & & & \\
\hline & High schol or lower level & 1,106 & 1,017 & 883 & 2,274 & 2,062 & 1,117 & 641 & 617 & 481 \\
\hline 6 & Hign school or lower level & 10.5 & 12.7 & 21 & 22.9 & 24.8 & 25.7 & 6.1 & 7.5 & 11.2 \\
\hline & Colleoe/ university deoree or hioher & 226 & 287 & 396 & 456 & 672 & 902 & 166 & 195 & 222 \\
\hline & College/ unıversity degree or higher & 18.4 & 18.3 & 13 & 41.1 & 40.7 & 28.3 & 13.7 & 12.3 & 7.1 \\
\hline & Setting & & & & & & & & & \\
\hline & Rural & 745 & 710 & 780 & 1,429 & 1,392 & 1,090 & 447 & 430 & 430 \\
\hline 7 & Rural & 12.1 & 14.1 & 20.2 & 24.4 & 27.1 & 27.6 & 7.3 & 8.5 & 11.1 \\
\hline & & 599 & 594 & 467 & 1,336 & 1,343 & 889 & 368 & 382 & 258 \\
\hline & Urban & 10.4 & 13 & 14.5 & 25.1 & 27.9 & 26.2 & 6.4 & 8.1 & 7.8 \\
\hline
\end{tabular}

was $16.5(\mathrm{SD}=3.9)$. The sample had the same rate in terms of sex and setting. There had been a decreasing trend over time amongst other variables, including being unmarried (91.4\% in round $1,84.4 \%$ in round 2 , and $72.8 \%$ in round 3 ), being student $(70.9 \%$ in round $1,66.0 \%$ in round 2 , and $41.7 \%$ in round 3 ), and having high school or lower level $(89.4 \%$ in round $1,83.4 \%$ in round 2 , and $57.9 \%$ in round 3 ). The poor and near-poor household had increase from $27.8 \%$ in round 1 to $38.2 \%$ in round 2 . For further details regarding demographic characteristics, see Table 1.

Prevalence of current smoking, harmful drinking and co-occurrence

The prevalence of current smoking among young people in CHILILAB increase significantly from $11.2 \%$ in round 1 (2006) to $17.6 \%$ in round 3 (2013). Similarly, the prevalence of harmful drinking amongst youth increase from $24.7 \%$ in round 1 (2006) to $26.8 \%$ in round 3 (2013). Across all demographic characteristics but not education level, there was a significant increase in the rate of current smoking among youth (Table 2). The current smoking rate shown a decrease rate among youth who attained higher education level (college/university degree or higher). Besides, three sub-groups of young people, that are, female, youth aged 20-24 years old, and youth having other works, had a stable smoking rate. In contrast to the rate of current smoking among youth, the trend rate of harmful drinking across all demographic characteristics, 
Table 3. Results of Logistic Regression Analysis for Smoking and Harmful Drinking By Demographic Characteristics: Three Rounds of Survey 2006-2013

\begin{tabular}{|c|c|c|c|c|c|c|c|c|c|c|}
\hline \multirow{2}{*}{\multicolumn{2}{|c|}{$\frac{\text { Variable }(\mathrm{OR} *)}{\text { Characteristics/ Year }}$}} & \multicolumn{3}{|c|}{ Smoking } & \multicolumn{3}{|c|}{ Harmful drinking } & \multicolumn{3}{|c|}{$\begin{array}{l}\text { Co-occurrence of smoking } \\
\text { and harmful drinking }\end{array}$} \\
\hline & & 2006 & 2009 & 2013 & 2006 & 2009 & 2013 & 2006 & 2009 & 2013 \\
\hline \multirow{2}{*}{\multicolumn{2}{|c|}{ Overall }} & 1,350 & 1,304 & 1,279 & 2,783 & 2,735 & 2,019 & 818 & 812 & 703 \\
\hline & & 13.6 & 17.6 & 24.7 & 27.5 & 26.8 & 7.1 & 8.3 & 9.5 & 23.1 \\
\hline \multirow{3}{*}{1} & Sex & & & & & & & & & \\
\hline & Male & Ref & Ref & Ref & Ref & Ref & Ref & Ref & Ref & Ref \\
\hline & Female & $0.04^{\mathrm{c}}$ & $0.01^{\mathrm{c}}$ & $0.02^{\mathrm{c}}$ & $0.43^{\mathrm{c}}$ & $0.38^{c}$ & $0.35^{\mathrm{c}}$ & $0.02^{\mathrm{c}}$ & $0.02^{\mathrm{c}}$ & $0.03^{\mathrm{c}}$ \\
\hline \multirow{4}{*}{2} & Age & & & & & & & & & \\
\hline & $10-14$ years old & Ref & Ref & Ref & Ref & Ref & Ref & Ref & Ref & Ref \\
\hline & 15-19 years old & $2.76^{c}$ & $2.13^{c}$ & $1.30^{c}$ & $1.94^{c}$ & $2.01^{\mathrm{c}}$ & $1.12^{\mathrm{c}}$ & $6.99^{c}$ & $2.85^{\mathrm{c}}$ & $1.40^{c}$ \\
\hline & 20-24 years old & $5.18^{c}$ & $2.96^{c}$ & $1.41 \mathrm{c}$ & $2.50 \mathrm{c}$ & $2.46 \mathrm{c}$ & $1.07^{\mathrm{c}}$ & $16.43^{\mathrm{c}}$ & $4.40^{c}$ & $1.77^{\mathrm{c}}$ \\
\hline \multirow{3}{*}{3} & Marital status & & & & & & & & & \\
\hline & Unmmarried & Ref & Ref & Ref & Ref & Ref & Ref & Ref & Ref & Ref \\
\hline & Married & 1.13 & 1.21 & $1.80^{b}$ & $0.59^{c}$ & $0.59^{c}$ & 0.86 & 0.87 & 0.89 & 1.33 \\
\hline \multirow{4}{*}{4} & Occupation & & & & & & & & & \\
\hline & Informal work & Ref & Ref & Ref & Ref & Ref & Ref & Ref & Ref & Ref \\
\hline & Pupils/ Students & $0.32^{\mathrm{c}}$ & $0.24^{\mathrm{c}}$ & $0.25^{\mathrm{c}}$ & 0.93 & 1.17 & 0.97 & $0.42^{\mathrm{c}}$ & $0.30^{\mathrm{c}}$ & $0.31^{\mathrm{c}}$ \\
\hline & Others & $0.74^{\mathrm{a}}$ & $0.58^{b}$ & $0.61^{b}$ & 0.98 & 1.08 & 0.98 & $0.70^{b}$ & $0.53^{\mathrm{c}}$ & $0.59^{b}$ \\
\hline \multirow{3}{*}{5} & Household income & & & & & & & & & \\
\hline & Poor and near poor & Ref & Ref & Ref & Ref & Ref & Ref & Ref & Ref & Ref \\
\hline & Middle or higher income & 0.85 & 0.91 & 0.86 & 1.11 & 0.97 & 0.87 & 0.9 & 0.9 & 0.93 \\
\hline \multirow{3}{*}{6} & Education level & & & & & & & & & \\
\hline & High school or lower level & Ref & Ref & Ref & Ref & Ref & Ref & Ref & Ref & Ref \\
\hline & College/ university degree or higher & 1.12 & 1.15 & 0.89 & $1.42^{\mathrm{c}}$ & 1.08 & $1.27^{\mathrm{a}}$ & $1.41^{\mathrm{b}}$ & 1.18 & 0.99 \\
\hline \multirow{3}{*}{7} & Setting & & & & & & & & & \\
\hline & Rural & Ref & Ref & Ref & Ref & Ref & Ref & Ref & Ref & Ref \\
\hline & Urban & 1.11 & $1.32^{\mathrm{a}}$ & 0.99 & 1.07 & 1.08 & 1.1 & 1.06 & $1.32^{\mathrm{a}}$ & 0.99 \\
\hline \multirow{3}{*}{8} & Current smoking & & & & & & & & & \\
\hline & No & - & - & - & Ref & Ref & Ref & - & - & - \\
\hline & Yes & - & - & - & $4.40^{c}$ & $3.83^{\mathrm{c}}$ & $5.05^{\mathrm{c}}$ & - & - & - \\
\hline \multirow{3}{*}{9} & Family members' smoking & & & & & & & & & \\
\hline & No & Ref & Ref & Ref & Ref & Ref & Ref & Ref & Ref & Ref \\
\hline & Yes & $1.56^{\mathrm{c}}$ & $1.34^{b}$ & $1.57^{\mathrm{c}}$ & 1.04 & 1.06 & 1.24 & $1.56^{\mathrm{c}}$ & $1.39^{b}$ & $1.81^{\mathrm{c}}$ \\
\hline \multirow{3}{*}{10} & Close-friends' smoking & & & & & & & & & \\
\hline & No & Ref & Ref & Ref & Ref & Ref & Ref & Ref & Ref & Ref \\
\hline & Yes & $2.81^{\mathrm{c}}$ & $3.91^{\mathrm{c}}$ & $2.59^{c}$ & $2.85^{\mathrm{c}}$ & $2.55^{\mathrm{c}}$ & $2.96^{c}$ & $5.83^{\mathrm{c}}$ & $6.13^{\mathrm{c}}$ & $4.54^{\mathrm{c}}$ \\
\hline \multirow{3}{*}{11} & Harmful drinking & & & & & & & & & \\
\hline & No & Ref & Ref & Ref & - & - & - & - & - & - \\
\hline & Yes & $3.73^{\mathrm{c}}$ & $3.15^{\mathrm{c}}$ & $2.14^{\mathrm{c}}$ & - & - & - & - & - & - \\
\hline \multirow{3}{*}{12} & Family members' drinking & & & & & & & & & \\
\hline & No & Ref & Ref & Ref & Ref & Ref & Ref & Ref & Ref & Ref \\
\hline & Yes & 1.14 & 1.17 & 1.14 & $1.47^{\mathrm{c}}$ & $1.54^{\mathrm{c}}$ & $1.43^{c}$ & $1.54^{\mathrm{c}}$ & $1.38^{b}$ & $1.59^{b}$ \\
\hline
\end{tabular}

*;a: represents statistically significant at $\mathrm{p}$-value $<0.05$; ${ }^{\text {b: }}$ represents statistically significant at $\mathrm{p}$-value $<0.01$; : represents statistically significant at p-value $<0.001$; OR: Odd ratio; Ref: Reference group

but youth aged 10-14 years old (age), youth being students and youth in poor or near-poor household (household income), were stable or lower over time.

The rate of co-occurrence of smoking and harmful drinking among youth also increased significantly from $7.1 \%$ in round 1 (2006) to $9.5 \%$ in round 3 (2013). Similar to current smoking among youth, the rate trend of co-occurring smoking and harmful drinking showed an increase trend among most of the demographic characteristics and their sub-groups over time. The rate of co-occurring smoking and drinking among poor and near-poor youth, for example, increased from $7.5 \%$ in round 1 (2006) to $12.1 \%$ in round 3 (2013). There were, however, 3 sub-groups of three variables, that are, young people aged 20-24 years old (age), having other works (occupation), having college/university degree or higher (education level), depicting a decrease trend in the rate of co-occurring smoking and harmful drinking.

Factors associated with current smoking, harmful drinking and co-occurrence

The primary logistic regression model depicted variables that were associated with current smoking, harmful drinking and co-occurrence of smoking and harmful drinking among youth (Table 3 ). Further, the final random-effects model confirmed the associated 
Table 4. Results of Random-Effects Logit Models for Smoking and Harmful Drinking by Demographic Characteristics: Three Rounds of Survey 2006-2013

\begin{tabular}{|c|c|c|c|c|c|c|c|c|c|c|}
\hline & Variable $\left(\mathrm{OR}^{*}\right)$ & Cur & rent smo & king & Harn & nful drin & aking & $\begin{array}{r}\text { Co-- } \\
\text { smoki }\end{array}$ & $\begin{array}{l}\text { occurren } \\
\text { ng and } h \\
\text { drinking }\end{array}$ & $\begin{array}{l}\text { ce of } \\
\text { armful }\end{array}$ \\
\hline & Characteristics & OR & $\begin{array}{c}\text { Lower } \\
\text { CI }\end{array}$ & $\begin{array}{c}\text { Upper } \\
\text { CI }\end{array}$ & OR & $\begin{array}{l}\text { Lower } \\
\text { CI }\end{array}$ & $\begin{array}{c}\text { Upper } \\
\text { CI }\end{array}$ & OR & $\begin{array}{c}\text { Lower } \\
\text { CI }\end{array}$ & $\begin{array}{c}\text { Upper } \\
\text { CI }\end{array}$ \\
\hline & Sex & & & & & & & & & \\
\hline 1 & Male & Ref & Ref & Ref & Ref & Ref & Ref & Ref & Ref & Ref \\
\hline & Female & $0.01^{\mathrm{c}}$ & 0.01 & 0.02 & $0.35^{\mathrm{c}}$ & 0.32 & 0.39 & $0.01^{\mathrm{c}}$ & 0.01 & 0.01 \\
\hline & Age & & & & & & & & & \\
\hline 2 & 10-14 years old & Ref & Ref & Ref & Ref & Ref & Ref & Ref & Ref & Ref \\
\hline 2 & $15-19$ years old & $2.43^{\mathrm{c}}$ & 1.95 & 3.03 & $1.91^{\mathrm{c}}$ & 1.7 & 2.14 & $3.44^{\mathrm{c}}$ & 2.69 & 4.42 \\
\hline & 20-24 years old & $5.03^{\mathrm{c}}$ & 3.72 & 6.8 & $2.61^{\mathrm{c}}$ & 2.18 & 3.11 & $9.10^{\mathrm{c}}$ & 6.53 & 12.7 \\
\hline & Marital status & & & & & & & & & \\
\hline 3 & Unmmarried & Ref & Ref & Ref & Ref & Ref & Ref & Ref & Ref & Ref \\
\hline & Married & 1.08 & 0.83 & 1.41 & $0.50^{\mathrm{c}}$ & 0.42 & 0.59 & $0.74^{\mathrm{a}}$ & 0.57 & 0.97 \\
\hline & Occupation & & & & & & & & & \\
\hline 4 & Informal work & Ref & Ref & Ref & Ref & Ref & Ref & Ref & Ref & Ref \\
\hline 4 & Pupils/ Students & $0.19^{c}$ & 0.16 & 0.24 & 0.99 & 0.85 & 1.14 & $0.27^{\mathrm{c}}$ & 0.21 & 0.34 \\
\hline & Others & $0.58^{\mathrm{c}}$ & 0.47 & 0.72 & 0.95 & 0.82 & 1.1 & $0.51^{\mathrm{c}}$ & 0.41 & 0.63 \\
\hline & Household income & & & & & & & & & \\
\hline 5 & Poor and near poor & Ref & Ref & Ref & Ref & Ref & Ref & Ref & Ref & Ref \\
\hline & Middle or higher income & 0.88 & 0.75 & 1.03 & 1.05 & 0.95 & 1.16 & 0.92 & 0.77 & 1.09 \\
\hline & Education level & & & & & & & & & \\
\hline 6 & High school or lower level & Ref & Ref & Ref & Ref & Ref & Ref & Ref & Ref & Ref \\
\hline & College/ university degree or higher & 1.05 & 0.84 & 1.3 & $1.31^{\mathrm{c}}$ & 1.15 & 1.5 & 1.25 & 0.99 & 1.56 \\
\hline & Current smoking & & & & & & & & & \\
\hline 7 & No & - & - & - & Ref & Ref & Ref & - & - & - \\
\hline & Yes & - & - & - & $4.40^{c}$ & 3.84 & 5.05 & - & - & - \\
\hline & Family members' smoking & & & & & & & & & \\
\hline 8 & No & Ref & Ref & Ref & Ref & Ref & Ref & Ref & Ref & Ref \\
\hline & Yes & $1.59^{\mathrm{c}}$ & 1.36 & 1.86 & $1.12 \mathrm{a}$ & 1.02 & 1.22 & $1.76^{\mathrm{c}}$ & 1.48 & 2.08 \\
\hline & Close-friends' smoking & & & & & & & & & \\
\hline 9 & No & Ref & Ref & Ref & Ref & Ref & Ref & Ref & Ref & Ref \\
\hline & Yes & $4.12^{\mathrm{c}}$ & 3.45 & 4.92 & $3.29^{\mathrm{c}}$ & 2.99 & 3.62 & $8.26^{\mathrm{c}}$ & 6.63 & 10.3 \\
\hline & Harmful drinking & & & & & & & & & \\
\hline 10 & No & Ref & Ref & Ref & - & - & - & - & - & - \\
\hline & Yes & $4.36^{c}$ & 3.71 & 5.13 & - & - & - & - & - & - \\
\hline & Family members' drinking & & & & & & & & & \\
\hline 11 & No & Ref & Ref & Ref & Ref & Ref & Ref & Ref & Ref & Ref \\
\hline & Yes & $1.18^{\mathrm{a}}$ & 1.01 & 1.38 & $1.55^{\mathrm{c}}$ & 1.41 & 1.7 & $1.64^{\mathrm{c}}$ & 1.38 & 1.94 \\
\hline
\end{tabular}

${ }^{a}$ represents statistically significant at p-value $<0.05$; brepresents statistically significant at p-value $<0.01$; crepresents statistically significant at p-value $<0.001$; OR, Odds ratio; CI, Confidence Interval; Ref, Reference group

variables with current smoking, harmful drinking and cooccurrence of smoking and harmful drinking among youth (Table 4). Notably, similar variables shared the significant association with smoking behaviour, harmful drinking and co-occurrence. Seven variables, that are, three demographic variables (sex, age, and occupation) and four tested variables regarding to family member and peer influences, had significant association with the smoking behaviour. Eight variables, consisting of four demographic variables (sex, age, marital status, and education level) and four tested variables regarding to family member and peer influences, significantly associated with this habit among youth. Finally, seven variables, that are, four demographic variables (sex, age, marital status and occupation) and three tested variables regarding to family member and peer influences, had significant association with co-occurring smoking and harmful drinking.

In terms of co-occurring smoking and harmful drinking among youth, the greatest likelihood of co-occurring smoking and harmful drinking was significantly associated with having close-friends who smoke $(\mathrm{OR}=8.26$, CI:6.63-10.28), older ages $(\mathrm{OR}=3.44-9.10)$, having family members who smoke (OR=1.76, CI:1.48-2.08), and having family members who use alcohol $(\mathrm{OR}=1.64$, CI:1.38-1.94). On the contrary, being female was the strongest protective factor against smoking and drinking $(\mathrm{OR}=0.00$, CI: 0.00-0.01). Being pupils or having other work was another protective factor. B eing female 
$(\mathrm{OR}=0.01, \mathrm{CI}: 0.01-0.01)$, being married $(\mathrm{OR}=0.74$, CI:0.57-0.97), and being students (OR=0.27, CI:0.21$0.51)$ or having other works $(\mathrm{OR}=0.51, \mathrm{CI}: 0.41-0.63)$ were associated with a lower likelihood of being current smoking and harmful drinking.

\section{Discussion}

We found a dramatically increase trend over time of co-occurring smoking and harmful drinking among a representative sample of 12,000 youth enrolled in a longitudinal survey in the CHILILAB in a northern province in Vietnam. The problems related to cooccurrence of smoking and harmful drinking among youth in Chi Linh could be considered to be representative for all youth in similar settings (peri-urban areas) in Vietnam.

The current study, to our best knowledge, is the first longitudinal analysis with regards to co-occurrence between smoking and harmful drinking among youth aged 10-24 years in Vietnam. Besides, our findings asserted the elevated trend of smoking rate $(11.2 \%$ to $17.6 \%)$ and harmful drinking rate $(24.7 \%$ to $26.8 \%)$, as well as the co-occurrence of smoking and harmful drinking $(7.1 \%$ to $9.5 \%$ ) among youth from round 1 to round 3 . This rate was lower than that of other studies on co-occurring of smoking and drinking in Vietnam and other countries (Johnson et al., 2000; Anderson, 2006; Nguyen et al., 2012). In United States, there was, for example, $34.8 \%$ of youth aged 18-24 years old co-occurring of smoking and drinking. Another study presented the rate of occurrence of smoking and drinking as high as $37 \%$ among migrant adolescents and young adults in Hanoi, Vietnam (Nguyen et al., 2012). This was explained because our study explored the co-occurring smoking and harmful drinking. Few current studies, to our best knowledge, have data about the prevalence of co-occurring smoking and harmful drinking.

The current study presented the four demographic characteristics (sex, age, marital status, and occupation) were associated with current smoking, harmful drinking, and co-occurring. Age and sex appeared to be the most crucial demographic variable for predicting the unhealthy behaviours regarding current smoking, harmful drinking, and co-occurring. Smoking and harmful drinking problems were significantly higher with being male, becoming older, having informal work, and being unmarried. Regarding family member and peer influences, young people who have close-friends who smoke had much higher risks $(8$ times) to be current smoking, having harmful drinking, and co-occurring. Besides, having family members who were smoking or drinking were also associated with higher risks of being current smoking, having harmful drinking, and co-occurring among young people. Finally, in terms of each smoking behaviour and harmful drinking habit, youth were significantly more likely to smoke when they had harmful drinking, and having harmful drinking when they smoked.

Our study highlighted that close-friends' smoking were the most important variable among all variables related to family member and peer influences to smoking and harmful drinking behaviours among youth. In consistent with our findings, there is accumulating evidence that peer and parental smoking and drinking increases the risk of adolescents' smoking initiation (Kobus, 2003; Yeh, 2006; Gilman et al., 2009). The behaviours of close-friends have been repeatedly stressed as the most important predictor of adolescent smoking and drinking (Kobus, 2003). The parental affects the likelihood of smoking, however, the influence declines overtime, whereas peer influence increases (den Exter Blokland et al., 2004; Otten et al., 2007). Young non-smokers who are friends with smokers and drinkers were more likely to initiate smoking and drinking than youth without smoking and drinking friends (Flay et al., 1994; Kobus, 2003; Yeh, 2006). Moreover, the current study repeatedly supported the positive association between current smoking and harmful drinking. The co-occurrence of cigarette smoking and harmful drinking among youth is frequently observed, and they are assumed to be highly correlated (mong youth is frequently observed, and they are assumed to be highly correlated (Johnson et al., 2000; Weitzman and Chen, 2005). In short, these findings suggested that anti-smoking and anti-drinking interventions should not be undertaken as stand-alone programs but integrated into one covering both the best friends and parents to maximize the outcomes.

Smoking, harmful drinking, and co-occurrence were especially common among male youth. Smoking and drinking have been repeatedly highlighted as the most pressing health problems in Vietnam but for males only (Nguyen et al., 2012; Bui et al., 2015). The concern for female smoking and drinking in South-east Asian countries, however, has been reported by a number of studies (Morrow and Barraclough, 2003; Hoang et al., 2010). The two main reasons for concern related to female smoking and drinking are the high absolute number of women who are effected and the rising smoking and drinking rate among women as they achieved more social and economic equality (Morrow and Barraclough, 2003). Further, gender-sensitive tobacco control policies are being challenged because of significant reduction of public health efforts among men but not among women (Morrow and Barraclough, 2003). In the current study, the smoking rate and harmful drinking rate among female tend to be stable (1.3\% and $13.7 \%$ in 2013 , respectively) but the co-occurrence of smoking and harmful drinking nearly double over time, from $0.4 \%$ in 2006 to $0.7 \%$ in 2013 . This implied that efforts to reduce the risky behaviours of smoking and drinking in Vietnam should be targeted not only at males but also females. Focusing on both sexes, we would be more likely to prevent a new epidemic of smoking and harmful drinking among women in Vietnam.

Our findings showed that the youth initiated smoking and harmful drinking at very early ages. We also found a substantial increase in the likelihoods of smoking and harmful drinking during the transition from adolescence to young adulthood. The risk of co-occurring smoking and harmful drinking among youth aged 20 years or higher, for example, were 9 times higher than that among youth aged 10-14. Consistent with our findings regarding smoking behaviour, previous studies reported that most habitual adult smokers initiated their habit before the age 
of 19 years ((Lantz et al., 2000; Morrow and Barraclough, 2003). Thus, anti-smoking and anti-drinking programs were particularly important to target youths in their early teen, that is, early adolescents (10-14 years old) or middle adolescents (15-17 years old). Although young people in their early teen have been the primary target of some antismoking and anti-drinking programs in several countries, most interventions in Vietnam targeted adults (15 years or higher) or older groups. Since early adolescents spend most of their time in school, school-based prevention programmes should be strengthened in Vietnam.

Smoking, harmful drinking, and co-occurrence were significantly higher among youth having informal work $(22.8 \%)$ than students $(5.4 \%)$ and youth having other works $(15.2 \%)$. Furthermore, the increase rate of occurrence of smoking and drinking among youth having informal work was quicker than other young groups. This finding agrees with other studies showing that unemployed young people have significantly higher levels of smoking and drinking than students (Johnson et al., 2000; Anderson, 2006). Combined all the above findings, our study highlighted the need for multi-level approaches aimed at smoking and harmful drinking reduction strategies. Interventions for smoking-control should not only focus on male adolescents (aged 10-19 years old) in school but also include all adolescents and those having informal work in the community. Consistent with our suggestion, school-based programmes and community interventions involving best friends, parents, mass media, and community organisations have been reported to have a stronger impact over time rather than stand-alone interventions (Lantz et al., 2000; Backinger et al., 2003).

Regarding methodological considerations, this study is strengthened by the use of a large sample, representative institutional sampling frame, and retrospective cohort. The co-occurrence of smoking and drinking among youth in Chi Linh could represent all youth in similar settings in Vietnam. Nevertheless, several limitations related to definition of health problems and loss of follow up deserve to be mentioned. Our definition of harmful drinking, those who reported that they had ever gotten into trouble because of drinking, could lead to higher prevalence rate of this event than other studies, which was defined as "harmful drinking as causing mental or physical damage" (National Institute for Health and Clinical Excellence, 2011). Regarding loss-of-follow up, we lost about 20-25\% of respondents in each following round. Because cooccurring smoking and drinking behaviour was a changed event, bias may have occurred in our study. However, with a large sample, we believed that this bias might have small impact on our analysis. Finally, findings from our study may not be generalized for youth beyond those living in extremely hard-to-reach areas and ethnic minority youth due to a very small proportion of the young people in these groups in our survey.

In conclusion, our findings indicate a dramatically increasing trend of smoking, harmful drinking and cooccurring among youth in a large-scale longitudinal survey in a northern province. The problems of smoking and drinking among youth in our study could represent all youth in peri-urban in Vietnam. Demographic characteristics (older age, being male, being unmarried, and having informal work) and the smoking and drinking status of the peer and family members, whereas closefriends' smoking was the most important variable, appeared to be predictors for smoking and drinking behaviour. This suggested that smoking and harmful drinking should not be solved with separate, stand-alone but integrated interventions.

\section{Acknowledgements}

We would like to thank collectors and the participants in this study in Chi Linh, a peri-urban district in Hai Duong province, Vietnam. This study was completed with financial support from the Ford Foundation. The authors declare that they have no competing interests.

\section{References}

Anderson P (2006). Global use of alcohol, drugs and tobacco. Drug Alcohol Rev, 25, 489-502.

Backinger CL, Fagan P, Matthews E, Grana R (2003). Adolescent and young adult tobacco prevention and cessation: current status and future directions. Tob Control, 12, 46-53.

Bui TV, Blizzard L, Luong KN, et al (2015). Declining Prevalence of Tobacco Smoking in Vietnam. Nicotine Tob Res, 17, 831-8.

den Exter Blokland EA, Engels RC, et al (2004). Lifetime parental smoking history and cessation and early adolescent smoking behavior. Prev Med, 38, 359-68.

Dierker L, Lloyd-Richardson E, Stolar M, et al (2006). The proximal association between smoking and alcohol use among first year college students. Drug Alcohol Depend, 81, 1-9.

Flay BR, Hu FB, Siddiqui O, et al (1994). Differential influence of parental smoking and friends' smoking on adolescent initiation and escalation of smoking. J Health Soc Behav, 35, 248-65.

Franceschi S, Talamini R, Barra S, et al (1990). Smoking and drinking in relation to cancers of the oral cavity, pharynx, larynx, and esophagus in northern Italy. Cancer Res, 50, 6502-7.

General Statistic Office of Vietnam (2015). The 1/4/2014 timepoint population change and family planning survey: Major findings. Hanoi: 2015.

Giang KB, Allebeck P, Spak F, Van Minh H, Dzung TV (2008). Alcohol use and alcohol consumption-related problems in rural Vietnam: an epidemiological survey using AUDIT. Subst Use Misuse, 43, 481-95.

Gilman SE, Rende R, Boergers J, et al (2009). Parental smoking and adolescent smoking initiation: an intergenerational perspective on tobacco control. Pediatrics, 123, 274-81.

Guindon GE, Georgiades K, Boyle MH (2008). Susceptibility to smoking among South East Asian youth: a multilevel analysis. Tob Control, 17, 190-7.

Jackson KM, Colby SM, Sher KJ (2010). Daily patterns of conjoint smoking and drinking in college student smokers. Psychol Addict Behav, 24, 424-35.

Johnson PB, Boles SM, Vaughan R, Kleber HD (2000). The cooccurrence of smoking and binge drinking in adolescence. Addict Behav, 25, 779-83.

Kobus K (2003). Peers and adolescent smoking. Addiction, 98 Suppl 1, 37-55.

Lantz P, Jacobson P, Warner K, et al (2000). Investing in youth 

tobacco control: a review of smoking prevention and control strategies. Tob Control, 9, 47-63.

Morrow M, Barraclough S (2003). Tobacco control and gender in south-east Asia. Part II: Singapore and Vietnam. Health Promot Int, 18, 373-80.

National Institute for Health and Clinical Excellence. Alcoholuse disorders: diagnosis, assessment and management of harmful drinking and alcohol dependence London: Canadian Institutes of Health Research, 2011 [cited 20164 Mar].

Nguyen LT, Rahman Z, Emerson MR, Nguyen MH, Zabin LS (2012). Cigarette smoking and drinking behavior of migrant adolescents and young adults in Hanoi, Vietnam. J Adolesc Health, 50, 61-7.

Otten R, Engels RC, van de Ven MO, Bricker JB (2007). Parental smoking and adolescent smoking stages: the role of parents' current and former smoking, and family structure. J Behav Med, 30, 143-54.

Schmid B, Hohm E, Blomeyer D, et al (2007). Concurrent alcohol and tobacco use during early adolescence characterizes a group at risk. Alcohol Alcohol, 42, 219-25.

Tran BH, Nguyen HT, Ho HT, Pham CV, Le VT, Le AV (2013). The Chi Linh Health and Demographic Surveillance System (CHILILAB HDSS). Int J Epidemiol, 42, 750-7.

Weitzman ER, Chen YY (2005). The co-occurrence of smoking and drinking among young adults in college: national survey results from the United States. Drug Alcohol Depend, 80, 377-86.

Witkiewitz K, Desai SA, Steckler G, et al (2012). Concurrent drinking and smoking among college students: An eventlevel analysis. Psychol Addict Behav, 26, 649-54.

Yeh MY (2006). Factors associated with alcohol consumption, problem drinking, and related consequences among high school students in Taiwan. Psychiatry Clin Neurosci, 60, 46-54.

Zheng TZ, Boyle P, Hu HF, et al (1990). Tobacco smoking, alcohol consumption, and risk of oral cancer: a case-control study in Beijing, people's republic of China. Cancer Causes Control, 1, 173-9. 